expands their societal impact. I believe that online learning will enable the creation of high-speed and possibly more efficacious multi- and interdisciplinary teaching environments around the world.

\section{CASE BY CASE}

As president of Arizona State University (ASU) - a large public research university that has four campuses in the Phoenix metropolitan area - I am optimistic about the potential for online learning to enable broader access to the highest levels of academic excellence. The ASU has embraced online learning and offers fully online undergraduate and graduate degree programmes. Faculty members are in charge of course content and complement online instruction with personal interaction. Interactive, adaptive learning platforms break down content and present it to students at an optimal pace and in a tailored sequence, while giving detailed feedback on performance to both students and professors. Our objective is to create an environment in which a person can learn anything, and thus we must avail ourselves of every tool, mechanism and means. The challenge remains for the ASU, like all universities, to evaluate the relevance of MOOCs to curricula and degree programmes and to determine whether and how to proceed with their implementation. This will inevitably advance on a case-by-case basis.
Regardless of any apprehension about online learning, there is now an opportunity to transcend the limits of the 'sage on the stage'. Online learning - including MOOCs - can enable and accelerate deeper or broader learning in certain subjects and thus

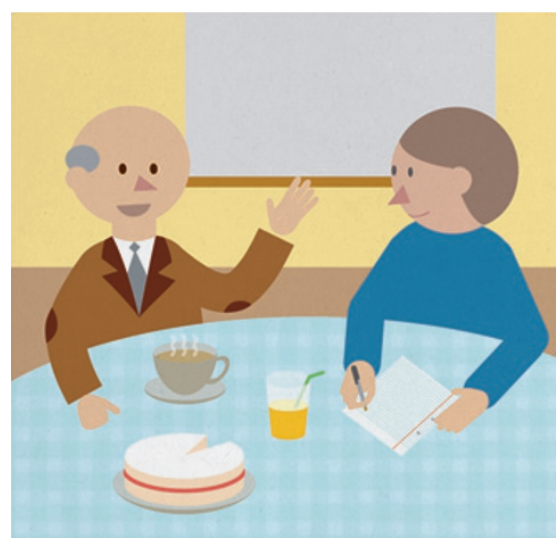

complement face-to-face or team-learning modalities. Where appropriate, MOOCs represent a simple, straightforward way to put quality content in front of lots of students at the same time, enabling scarce resources to be deployed where they are most needed: in the creation of teaching, learning and discovery environments that can educate millions to the levels necessary to ensure successful and continued adaptation in the future.
One cautionary note should be sounded: to the naive, MOOCs seem like the quick fix for cost containment - itself a crucial objective if there is to be any social equity in the higher-education system. A professor from an exclusive university, such as Columbia, could have his or her course put onto the Web as a MOOC, then students at larger public universities could take that course instead of its equivalent on their home campuses, thereby shrinking faculties and costs. But producing a master adaptive learner will always require interaction with the creative and caring teacher-scholars known as the faculty. The integration of interactive online learning tools such as MOOCs can be useful, but these disembodied teaching vehicles are no substitute for teacher interaction.

I can only hope that in our enthusiasm for MOOCs, we look before we leap into a dystopia in which the masses are taught by robots, and the gifted and wealthy by living, breathing professors. We need to end up in a world in which technology once and for all makes higher education more accessible by lowering its cost and enhancing its impact across our great and highly diverse society.

Michael M. Crow is president of Arizona State University, Tempe, Arizona 85287, USA. He previously served as executive vice-provost and professor of science policy at Columbia University in New York. e-mail:michael.crow@asu.edu

\title{
Online on-ramps
}

\section{Giving scientists greater access to conceptual and technical tuition through massive open online courses will aid interdisciplinary research, say Hazel Sive and Sanjay Sarma.}

$\mathrm{T}$ here has been a lot of discussion about the way in which massive open online courses (MOOCs) might change the landscape of education. A transformation is likely, but in a form more nuanced than current conversations imply (see go.nature. com/cynqvp).

Another question has had less attention: how will MOOCs affect research and scholarly enterprise at universities? Will the courses lead to a decline in the amount or quality of study? Or will a huge increase in online learning improve research?

We are both deeply involved in the MOOC culture at the Massachusetts Institute of Technology (MIT) in Cambridge. We are members of the university's Task Force on the Future of MIT Education, which focuses on the use and effect of MOOCs, and we both run active research groups, so we appreciate the dual tugs of teaching and scholarship. Our opinion is that, at least for residential, research-focused universities, the main impact of MOOCs on research culture will be to aid interdisciplinary work by enabling greater access to conceptual or technical tuition.

Many factors influence scholarship and research in a university. These include funding, space, equipment, research collaborations, the number of faculty members, the

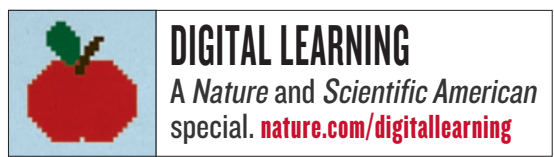

presence of faculty members on campus and the time available for research. Research is often deeply affected by the insight and questioning of smart undergraduate students or postdocs. Fields move forwards through the ability to think in novel, cross-disciplinary ways, and through familiarity with state-ofthe-art techniques.

Which parts of that equation will MOOCs affect? A common view is that they will replace professors. Clearly, this would diminish the volume of research and stifle the collegial discourse that fosters collaboration and original thinking. We do not consider this to be a likely outcome - except perhaps in parts of the world where students have limited access to quality education and where a MOOC might stand in for 


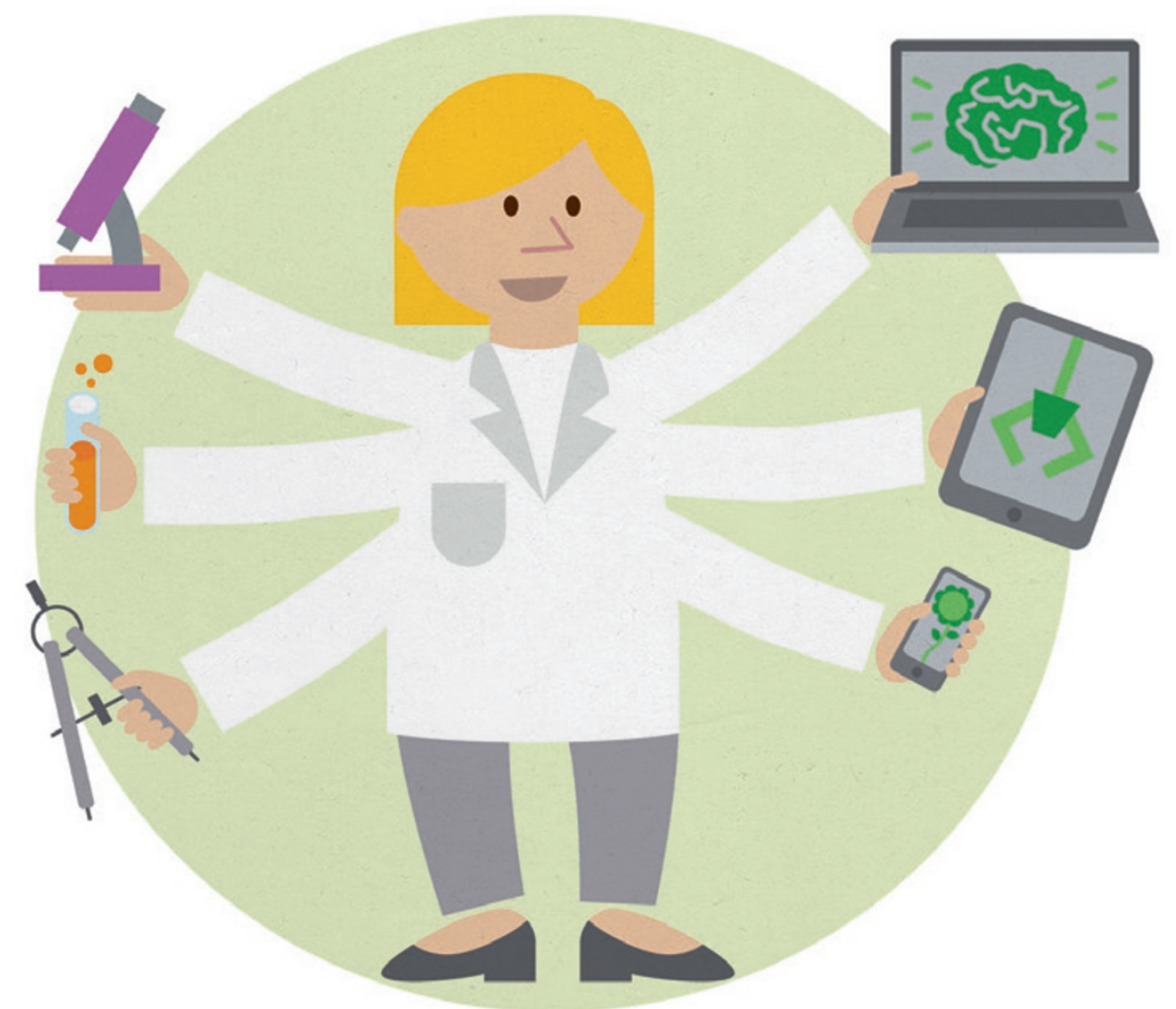

face-to-face teaching, possibly leading to a cut in faculty numbers. Data on this point are not yet available owing to the newness of these teaching tools.

In research-intensive universities, it is our experience that the time in a faculty member's schedule that is freed up through the use of online material is invested back into 'flipped' classrooms - students have already studied lecture material online, meaning that class time is used for discussion, hands-on activities, problem solving and research. Similarly, cutting-edge graduate classes thrive on faculty-student interaction. Any reduction in lecture time is reinvested in the classroom to make the experience more comprehensive and fruitful for graduate students.

When online learning tools were used to teach electrical engineering to MIT undergraduates, faculty time that would have been devoted to lecturing went back into the course as alternative ways of teaching the material. These included practical demonstrations, hands-on experiments and interactive laboratory sessions featuring design, creation, debugging, small group discussions and one-to-one student-instructor feedback.

The increase in problem solving and discussion could expose more undergraduates to research questions and strategy earlier and more extensively than at present. This could encourage undergraduates to pursue research during their degree period or beyond, thereby enriching research culture.

For faculty members devising MOOCs and developing additional learning tools, there might be a short-term dip in research productivity. Such a dip is experienced by any devoted teacher who devises a new course, but the high-tech nature of MOOCs can involve a bigger time investment than normal. Our colleagues at MIT report that developing a new MOOC takes between half a semester and a full one, which lasts about 14 weeks.

The most important impact on research will probably be interdisciplinary online education. Say that an MIT student needs to learn current techniques in machine learn-

ing to apply to her

"Online main area of study, education is mechanical engineerunlikely to ing. An appropriate class is not offered at dentfaculty MIT until the next engagement in spring, but the Uniresearch." versity of California, Berkeley, offers an online course that she can take this summer. She takes the course and an adviser at MIT vets her progress. By the autumn, the student is ready to apply her newly acquired skills to her thesis research.

A related and crucial benefit of MOOCs to research stems from modules that will help investigators to straddle fields by aiding fluid learning. Modules can range from, for example, a 10-minute video segment to the content of multiple full-length lectures. Thus, for example, a postdoctoral neuroscientist might need to learn how to measure changes in cell shape. A full course is not necessary. Multiple modules explaining relevant concepts are available online from several universities. The postdoc uses these to increase his competency faster, more cheaply and more comprehensively than he would by taking a course, visiting a relevant laboratory or attending a meeting. This sort of learning has the potential to improve research output.

The serious decline of research funding in the United States and in other parts of the world is reducing the ability of researchers to travel to acquire training in person. Although we in no way suggest that virtual education can entirely plug this gap, it can help scientists to gain some of the conceptual and technical expertise that is necessary to advance research.

Although we feel that online education is unlikely to dent faculty engagement in research, it will be important to monitor carefully the amount of time that faculty members spend on developing MOOCs. We suggest that this is done at the departmental level to ensure that a push for online educational development is balanced by responsiveness to any research challenges that result.

Hazel Sive is professor of biology and associate dean of science at the Massachusetts Institute of Technology (MIT) in Cambridge, Massachusetts, USA. She is also at the Whitehead Institute for Biomedical Research, Cambridge, Massachusetts. Sanjay Sarma is professor of mechanical engineering and director of digital learning at MIT.

e-mails: sive@wi.mit.edu; sesarma@mit.edu 